\title{
Comparison of different Clinical Metrology Instruments in dogs with osteoarthritis
}

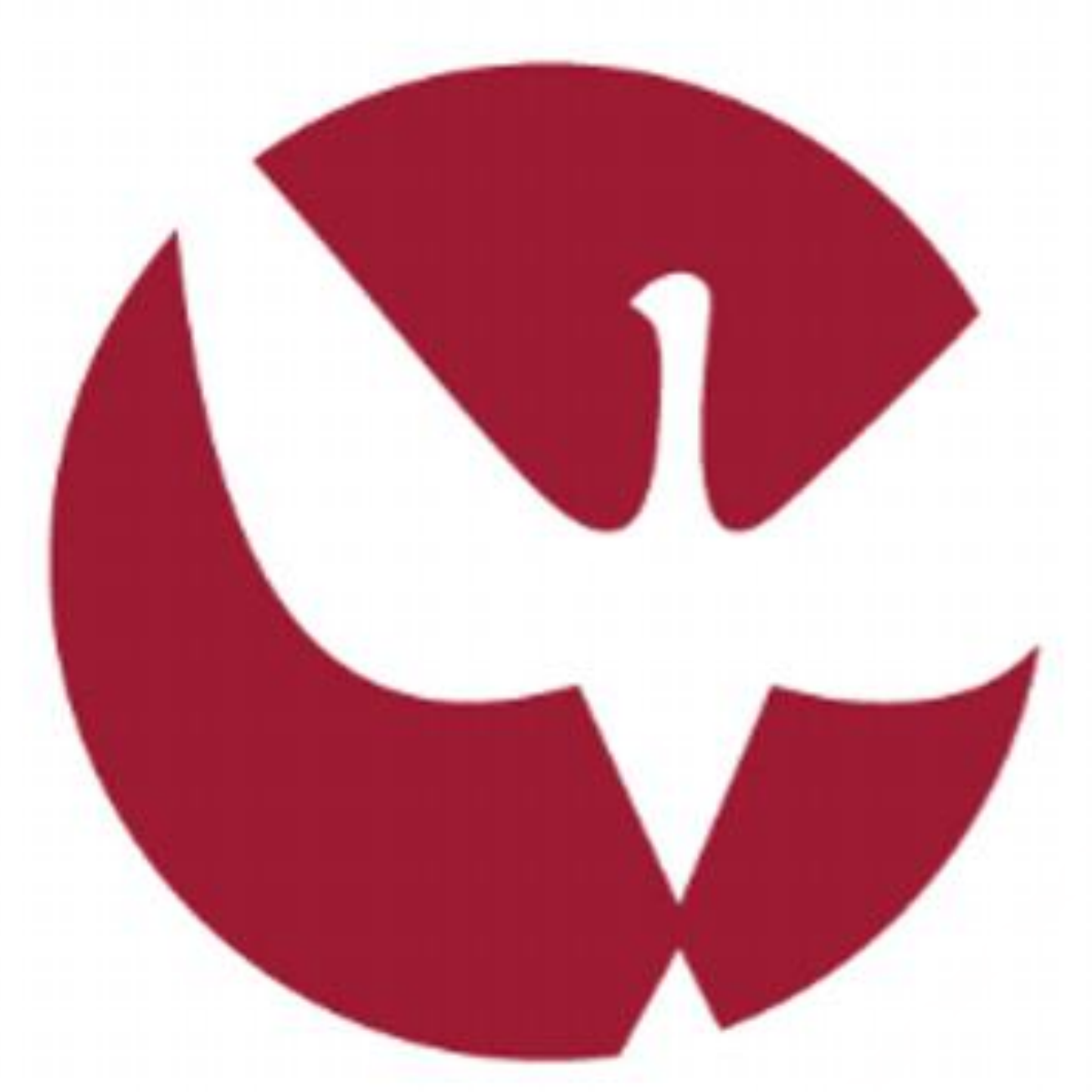

\author{
J. C. Alves ${ }^{1,2, *}$; A. Santos $;$; P. Jorge'; C. Lavrador² ;. M. Carreira ${ }^{3,4}$
}

'Guarda Nacional Republicana; ${ }^{2}$ MED - Mediterranean Institute for Agriculture, Environment and Development, Universidade de Évora; ${ }^{3}$ Interdisciplinary Centre for Research in Animal Health (CIISA) - University of Lisbon, (FMV/ULisboa) - Portugal. ${ }^{4}$ Anjos of Assis Veterinary Medicine Centre; *alves.jca@gnr.pt

To test construct and criterion validity of four Clinical Metrology Instruments (CMIs) in dogs with bilateral hip osteoarthritis.

\section{MATERIALS AND METHODS:}

Fifty police working dogs were evaluated, using a stance analyzer in six different moments, over a 180-day period;

Weight-bearing symmetry index and deviation from normal weight-bearing were calculated and used as criterion validity';

Three hundred responses to the Canine Brief Pain Inventory (CBPI, divided in pain severity score - PSS and pain interference score - PIS, the Liverpool Osteoarthritis in Dogs (LOAD), the Canine Orthopedic Index (COI, with four domains: stiffness, gait, function and quality of life - $\mathrm{OOL}$ ) and the Hudson Visual Analogue Scale, were compared;

Correlations between CMIs were evaluated as construct validity. Internal consistency was tested with Cronbach's $\alpha^{1}$.

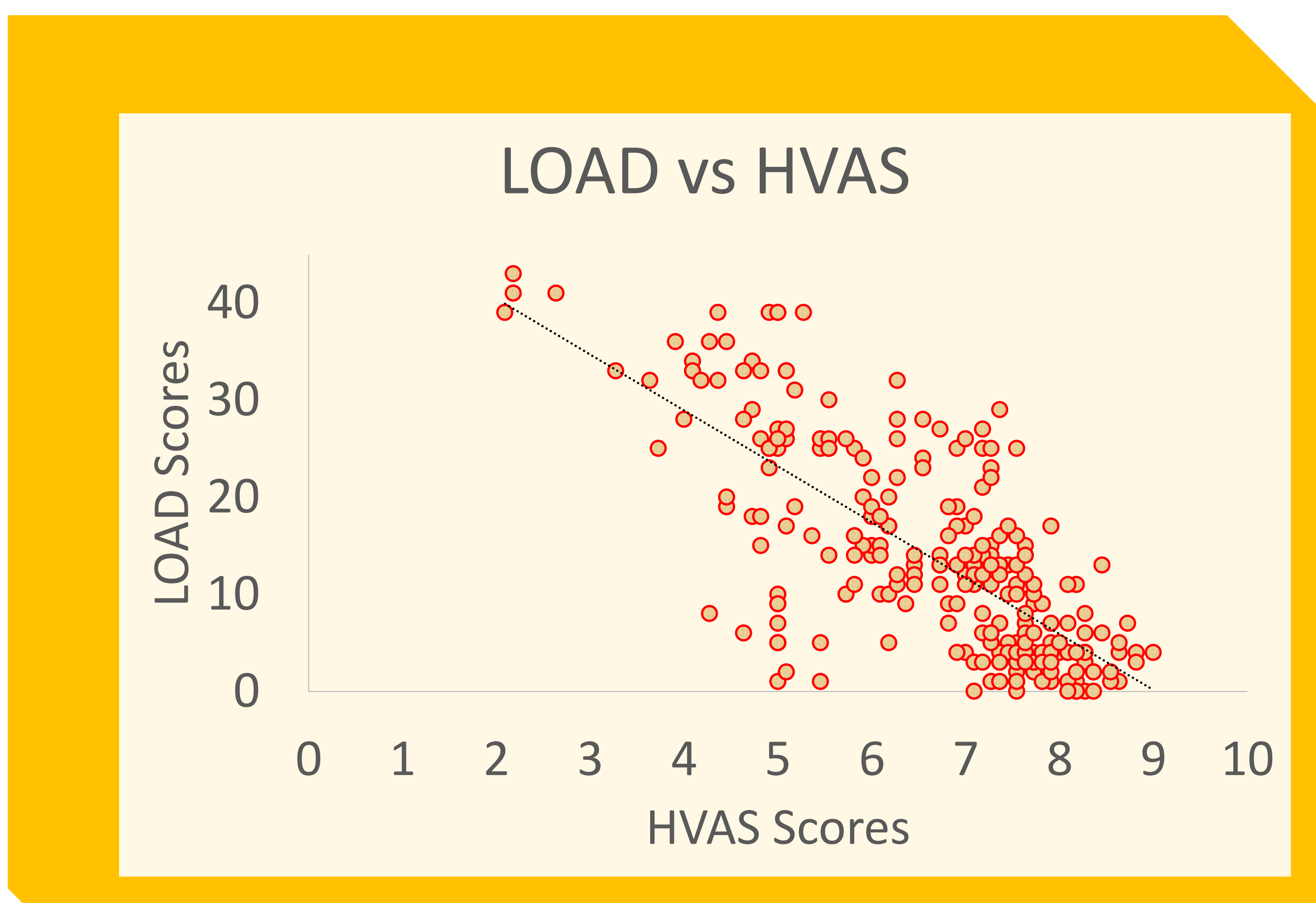

Fig. 1 - Scatterplot of LOAD and HVAS.

\section{RESULTS:}

Significant weak correlation was found between all CMls and stance analysis symmetry index measure and deviation, indicating criterion validity;

Cronbach's a was 0.80 for HVAS, 0.98 for CBPI, 0.97 for LOAD and 0.98 for COl;

HVAS showed a negative correlation with all other scores, particularly PSS (-0.81) and PIS (-0.83);

PSS and PIS showed a good correlation with all other scores, particularly LOAD (0.80 and 0.82) and COI (0.80 and 0.82).

LOAD scores had high correlation with all scores, especially COI (0.91);

COI dimensions showed good correlation comparing one to another.

\section{CONCLUSTONS:}

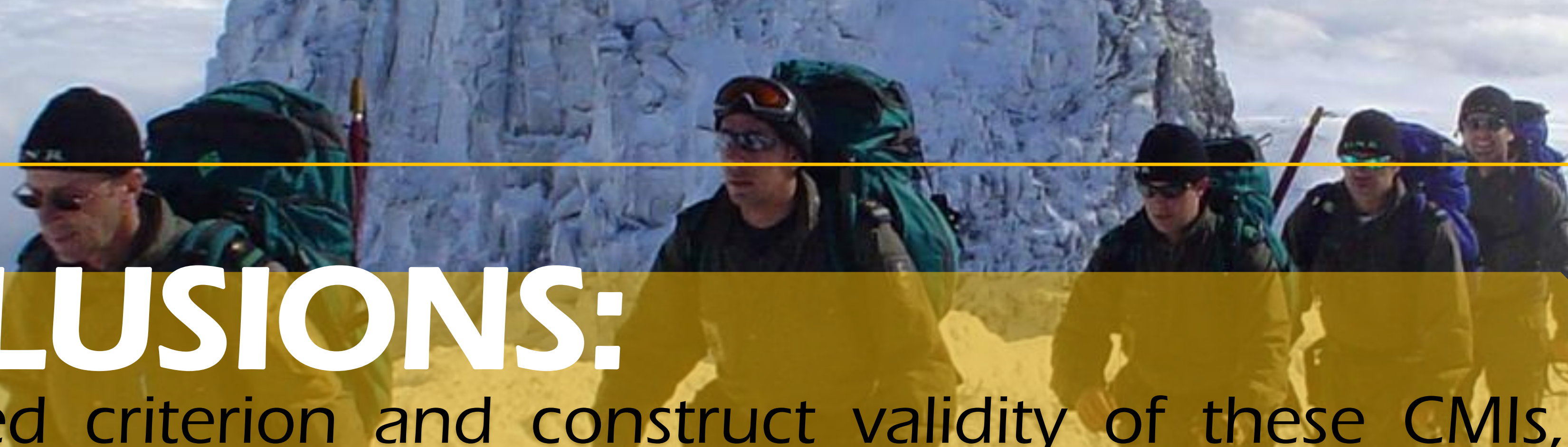

We presented criterion and construct validity of these CMls, which are able to capture various dimensions of $\mathrm{OA}$.

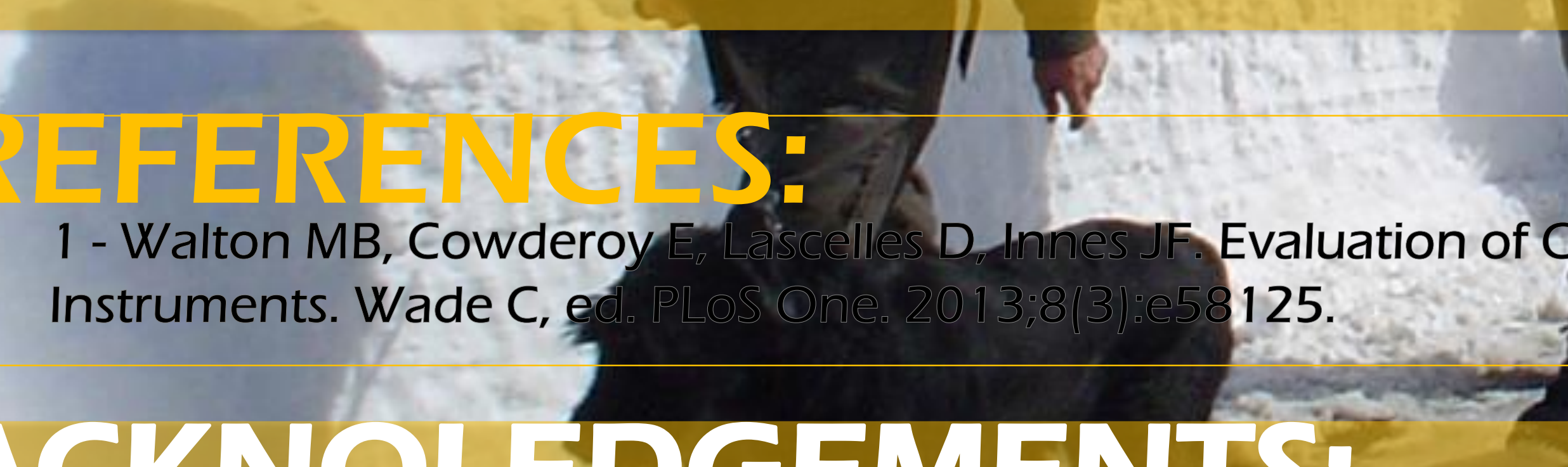

ACKNOLEDGEMENTS:

The authors would like to thank Companion, LiteCure LLC, and Concessus, S.A., for providing the digital thermography camera used in this study. 